\title{
On the coherence of dissipative soliton resonance square pulses
}

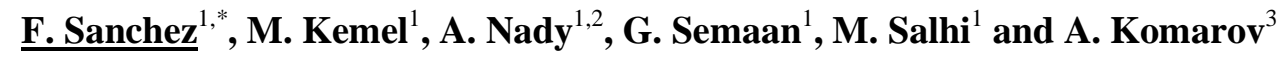 \\ ${ }^{1}$ Laboratoire de Photonique d'Angers, Faculté des Sciences, 2 Bd Lavoisier, 49045 Angers Cedex \\ ${ }^{2}$ Department of Physics, Faculty of Sciences, Beni-Suef University, 62511 Beni-Suef, Egypt \\ ${ }^{3}$ Institute of Automation and Electrometry, SB RAS, Novosibirsk, Russia \\ *E-mail: francois.sanchez@univ-angers.fr
}

DOI: 10.31868/RFL2020.86

Square-pulses in passively mode-locked fiber lasers are generally considered to be the manifestation of the dissipative soliton resonance (DSR). The DSR is a particular solution of the nonlinear propagation equation and it does not suffer from any wavebreaking as it was first predicted in [1,2]. Consequently both the energy and the width of the pulses increase linearly with the pumping power while the peak power remains clamped to a fixed value. In addition, the optical spectrum remains invariant versus the pumping power. Numerous experimental papers reported such kind of behavior and concluded that the observed pulses were real DSR pulses [3-5].

Another important characteristic of DSR pulses is that they exhibit square shape with a perfect temporal coherence. This characteristic is not easy to check because of the pulse duration in square-pulse regime which ranges from few to hundreds of nanoseconds [3-5]. In fact, the coherence measurement in square-pulse regime has been done only with ultra-short pulses in the ps range [3]. In such case it was demonstrated that the pulses were effectively coherent thus confirming that they were real DSR pulses.

In this communication we investigate the coherence of square-pulses in a passively mode-locked double-clad fiber laser and verify whether they operate in DSR regime or not. We demonstrate that although almost all the characteristics of DSR pulses are verified (energy and width scaling with pump power, peak power clamping, optical spectrum), the square-pulses are not temporally coherent thus demonstrating that square-pulses are not necessarily DSR pulses. Our conclusion is based on several series of experiment with distinct optical cavities operating in square-pulse regime in the nanosecond range. The experimental setups consist of two different methods of measurement. The first one is based on a Mach-Zehnder interferometer with a fixed delay which allows to scan a small part of a long square pulse. The second method is based on the Dispersive Fourier Transform (DFT) technique which allows to directly conclude on the pulse coherence.

\section{References}

[1] A. Komarov, H. Leblond and F. Sanchez, Phys. Rev. A 71, 053809 (2005).

[2] W. Chang, A. Ankiewicz et al, Phys. Rev. A 78, 023830 (2008).

[3] X. Wu, D. Y. Tang et al, Opt. Express 17, 5580 (2009).

[4] K. Krzempek, Opt. Express 23, 030651 (2015).

[5] G. Semaan, F. Ben Braham et al, Opt. Lett. 20, 4767 (2016). 